\title{
Linear Approximations of Nonlinear FIR Systems for Separable Input Processes
}

\author{
Martin Enqvist, Lennart Ljung \\ Division of Automatic Control \\ Department of Electrical Engineering \\ Linköpings universitet, SE-581 83 Linköping, Sweden \\ WWW: http://www. control.isy.liu.se \\ E-mail: maren@isy.liu.se, ljung@isy.liu.se
}

29th December 2005

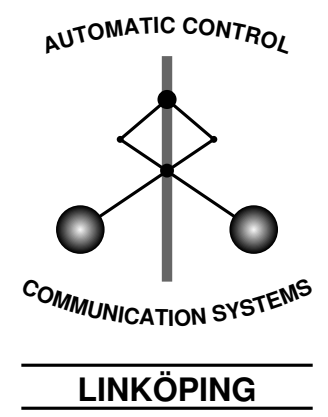

Report no.: LiTH-ISY-R-2718

Submitted to Automatica

Technical reports from the Control \& Communication group in Linköping are available at http://www. control.isy.liu.se/publications. 


\begin{abstract}
Nonlinear systems can be approximated by linear time-invariant (LTI) models in many ways. Here, LTI models that are optimal approximations in the meansquare error sense are analyzed. A necessary and sufficient condition on the input signal for the optimal LTI approximation of an arbitrary nonlinear finite impulse response (NFIR) system to be a linear finite impulse response (FIR) model is presented. This condition says that the input should be separable of a certain order, i.e., that certain conditional expectations should be linear. For the special case of Gaussian input signals, this condition is closely related to a generalized version of Bussgang's classic theorem about static nonlinearities. It is shown that this generalized theorem can be used for structure identification and for identification of generalized Wiener-Hammerstein systems.
\end{abstract}

Keywords: System identification, Mean-square error, Nonlinear systems, Linearization 


\title{
Linear Approximations of Nonlinear FIR Systems for Separable Input Processes
}

\author{
Martin Enqvist, Lennart Ljung
}

2005-12-29

\begin{abstract}
Nonlinear systems can be approximated by linear time-invariant (LTI) models in many ways. Here, LTI models that are optimal approximations in the mean-square error sense are analyzed. A necessary and sufficient condition on the input signal for the optimal LTI approximation of an arbitrary nonlinear finite impulse response (NFIR) system to be a linear finite impulse response (FIR) model is presented. This condition says that the input should be separable of a certain order, i.e., that certain conditional expectations should be linear. For the special case of Gaussian input signals, this condition is closely related to a generalized version of Bussgang's classic theorem about static nonlinearities. It is shown that this generalized theorem can be used for structure identification and for identification of generalized Wiener-Hammerstein systems.
\end{abstract}

\section{Introduction}

Nonlinear systems are often approximated using linear models. For example, local approximations around a set point can normally be obtained by differentiating a mathematical description of a nonlinear system. Typically, such approximations are only useful in an operating region around the set point and they can be hard to obtain if the nonlinearity is unknown. An alternative can be to derive a linear approximation that models the behavior of the nonlinear system for a particular input signal. This is the type of approximation that is studied in this paper.

More specifically, we consider single input single output (SISO) nonlinear systems with inputs $u(t)$ and outputs $y(t)$ that are stationary stochastic processes. For a such a system, the linear time-invariant (LTI) model that minimizes the mean-square error $\mathrm{E}\left((y(t)-G(q) u(t))^{2}\right)$ with respect to all stable and causal models $G(q)$ is analyzed. Here, $q$ denotes the shift operator, $q u(t)=u(t+1)$, and $\mathrm{E}(x)$ denotes the expected value of the random variable $x$. The mean-square error optimal model is here called the output error linear time-invariant second order equivalent (OE-LTI-SOE).

Since an OE-LTI-SOE of a nonlinear system is derived for a particular pair of input and output processes, the OE-LTI-SOE will usually be input dependent. Furthermore, in general a nonlinear finite impulse response (NFIR) system will not have a finite impulse response (FIR) OE-LTI-SOE. However, if a certain type of input signals is used, the OE-LTI-SOE will be an FIR model. 
The main result of this paper is a necessary and sufficient condition on the input signal for the OE-LTI-SOEs of all NFIR systems in a wide class of systems to be FIR models. This result, which is presented in Theorem 3.1, is an extension to NFIR systems of a similar result for static nonlinearities (Nuttall, 1958). More specifically, Nuttall (1958) presents a useful condition on the input for the property

$$
R_{y u}(\tau)=b_{0} R_{u}(\tau)
$$

to hold for an arbitrary static nonlinearity. Here, $R_{y u}(\tau)=\mathrm{E}(y(t) u(t-\tau))$ is the cross-covariance function between output and input and $R_{u}(\tau)=\mathrm{E}(u(t) u(t-\tau))$ is the covariance function of the input. It turns out that (1) holds for any static nonlinearity in a wide class of functions if and only if the input signal is separable. Separability of a process in Nuttall's sense means that the conditional expectation $\mathrm{E}(u(t-\sigma) \mid u(t))$ satisfy

$$
\mathrm{E}(u(t-\sigma) \mid u(t))=c(\sigma) u(t),
$$

where $c(\sigma)=R_{u}(\sigma) / R_{u}(0)$. In Nuttall (1958), a number of signals that have this property are listed, e.g. Gaussian processes, sine wave processes and phase modulated processes. In addition, McGraw and Wagner (1968) have shown that signals with elliptically symmetric distributions are separable and they have also characterized these signals further.

The notion of separable processes is related to Bussgang's classic theorem about Gaussian signals (see Bussgang (1952) or, for example, Papoulis (1984)).

\section{Theorem 1.1 (Bussgang)}

Let $y(t)$ be the stationary output from a static nonlinearity $f$ with a stationary Gaussian input $u(t)$, i.e., $y(t)=f(u(t))$. Assume that the expectations $\mathrm{E}(y(t))=\mathrm{E}(u(t))=0$. Then

$$
R_{y u}(\tau)=b_{0} R_{u}(\tau), \quad \forall \tau \in \mathbb{Z}
$$

where $R_{y u}(\tau)=\mathrm{E}(y(t) u(t-\tau)), R_{u}(\tau)=\mathrm{E}(u(t) u(t-\tau))$ and $b_{0}=\mathrm{E}\left(f^{\prime}(u(t))\right)$.

Besides Nuttall (1958), Bussgang's theorem has been extended to other classes of signals than Gaussian by Barrett and Lampard (1955) and Brown (1957). It has also been extended to NFIR systems (see, for example, Scarano et al., 1993).

In this paper, we restate the extended version of Bussgang's theorem for NFIR systems with Gaussian inputs. Furthermore, we show some new results about how this theorem can be used for structure identification of NFIR systems or for identification of generalized Wiener-Hammerstein systems. Such systems consist of three subsystems, first an LTI system followed by an NFIR system and after that another LTI system. Similar results have previously been presented for Wiener-Hammerstein systems where the nonlinear block is static (Billings and Fakhouri, 1982; Korenberg, 1985; Bendat, 1998).

The main purpose of this paper is to analyze some asymptotic properties of linear model estimates obtained by system identification using input and output data from nonlinear systems. The system identification method that is studied here is the prediction-error method (Ljung, 1999), and we will only investigate its asymptotic behavior when the number of measurements tends to infinity. 
A general, parameterized LTI model can be written

$$
y(t)=G(q, \theta) u(t)+H(q, \theta) e(t),
$$

where $G(q, \theta)$ describes how the input signal $u(t)$ affects the system output $y(t)$ and $H(q, \theta)$ describes the influence of the white noise $e(t)$ and where $\theta$ is a parameter vector. The parameters can, for example, be the coefficients of the numerator and denominator polynomials of $G(q, \theta)$ and $H(q, \theta)$ if these functions are rational. The main idea in prediction-error methods is to compare the measured true system output with output predictions based on (2) using, for example, a quadratic criterion. By minimizing this criterion with respect to $\theta$, parameter estimates are found.

It can be shown (Ljung, 1978) that the prediction-error parameter estimate under rather general conditions will converge to the parameters that minimize a mean-square error criterion $\mathrm{E}\left(\left(H^{-1}(q, \theta)(y(t)-G(q, \theta) u(t))\right)^{2}\right)$. With this result in mind, it is obvious that the results in this paper explains asymptotic properties about the prediction-error parameter estimate in the special case when $H(q, \theta)=1$.

However, the existence of a mean-square error optimal LTI approximation does not imply that the parameters in a parameterized model will always converge to values that correspond to the optimal model. Of course, this can only happen if the chosen model structure contains the OE-LTI-SOE. If a parameterized model of lower order than the OE-LTI-SOE is used, the parameters will converge to values that give an as good approximation of the optimal model as possible for the particular input signal that has been used. Such approximations of the OE-LTI-SOE are discussed in Section 2.

LTI approximations of nonlinear systems are discussed also by Pintelon and Schoukens (2001). They use the term related linear system for the mean-square error optimal LTI approximation and view the part of the output signal that this model cannot explain as a nonlinear distortion. Relevant material can be found also in Pintelon et al. (2001), Pintelon and Schoukens (2002) and Schoukens et al. (2003). Schoukens et al. (2004) have also discussed benefits and drawbacks of different input signals for LTI approximations.

The idea of deriving an LTI approximation by differentiation of a nonlinear system is used, for example, by Mäkilä and Partington (2003). They study LTI approximations of nonlinear systems for $l^{\infty}$-signals and use the notion of Fréchet derivatives to derive some of the approximations. Related material can be found in Partington and Mäkilä (2002), Mäkilä (2003a), Mäkilä (2003b) and in Mäkilä and Partington (2004). LTI approximations for deterministic signals are also discussed in Sastry (1999) and in Horowitz (1993).

\section{Output Error LTI-SOEs}

In this paper, we will only consider nonlinear systems with input and output signals that have certain properties. These signal assumptions are listed here.

Assumption A1. Assume that

(i) The input $u(t)$ is a real-valued stationary stochastic process with

$$
\mathrm{E}(u(t))=0 .
$$




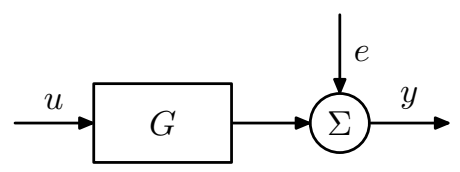

Figure 1: The output error model.

(ii) There exist $K>0$ and $\alpha, 0<\alpha<1$ such that the second order moment $R_{u}(\tau)=\mathrm{E}(u(t) u(t-\tau))$, satisfies

$$
\left|R_{u}(\tau)\right|<K \alpha^{|\tau|}, \quad \forall \tau \in \mathbb{Z} .
$$

(iii) The z-spectrum $\Phi_{u}(z)$ (i.e., the z-transform of $R_{u}(\tau)$ ) has a canonical spectral factorization

$$
\Phi_{u}(z)=L(z) r_{u} L\left(z^{-1}\right),
$$

where $L(z)$ and $1 / L(z)$ are causal transfer functions that are analytic in $\{z \in \mathbb{C}:|z| \geq 1\}, L(+\infty)=1$ and $r_{u}$ is a positive constant.

Assumption A2. Assume that

(i) The output $y(t)$ is a real-valued stationary stochastic process with

$$
\mathrm{E}(y(t))=0 .
$$

(ii) There exist $K>0$ and $\alpha, 0<\alpha<1$ such that the second order moments $R_{y u}(\tau)=\mathrm{E}(y(t) u(t-\tau))$ and $R_{y}(\tau)=\mathrm{E}(y(t) y(t-\tau))$ satisfy

$$
\begin{aligned}
\left|R_{y u}(\tau)\right|<K \alpha^{|\tau|}, \quad \forall \tau \in \mathbb{Z}, \\
\left|R_{y}(\tau)\right|<K \alpha^{|\tau|}, \quad \forall \tau \in \mathbb{Z} .
\end{aligned}
$$

In Assumptions A1(i) and A2(i) it is required that both the input and the output signal have zero mean. In practice, this assumption does not exclude systems with input and output signals that vary around a nonzero set point from being analyzed using the results in this paper. For such a system, it is always possible to define new input and output signals that describe the deviations from the set point by subtracting the corresponding means of the two signals. By their construction, these new signals will have zero mean and they will hence satisfy the zero mean assumption in this paper.

As mentioned above, we will consider here only models where the noise description $H$ is fixed to 1, i.e., output error models (Ljung, 1999). The structure of an output error model is shown in Figure 1.

Besides the restriction to output error models, some assumptions concerning the two basic system properties causality and stability will also be used in this paper. For the sake of completeness, the definitions used in these assumptions are included here. First, the notion of a causal or anticausal sequence will be defined.

Definition 2.1. A sequence $(m(k))_{k=-\infty}^{\infty}$ is causal if $m(k)=0$ for all $k<0$ and strictly causal if $m(k)=0$ for all $k \leq 0$. The sequence is anticausal if $m(k)=0$ for all $k>0$ and strictly anticausal if $m(k)=0$ for all $k \geq 0$. 
The notion of causality can be used also for LTI systems or models:

Definition 2.2. An LTI system or model is (strictly) causal if its impulse response is (strictly) causal. Similarly, an LTI system or model is (strictly) anticausal if its impulse response is (strictly) anticausal.

In some cases, we will need to extract the causal part of a noncausal system. This will be done using the following notation.

$$
[G(z)]_{\text {causal }}=\left[\sum_{k=-\infty}^{\infty} g(k) z^{-k}\right]_{\text {causal }}=\sum_{k=0}^{\infty} g(k) z^{-k}
$$

Causality of an LTI system implies that the system output only depends on past and present values of the input signal. Since all real-life systems are causal and we want LTI models that resemble the corresponding systems as much as possible, we will thus only consider causal models here. Note that all results in this paper can be reformulated, with obvious changes, for strictly causal models if such are desired.

Another important property of LTI systems is stability. In this paper, we will only use the type of stability called bounded input bounded output stability, which is defined as follows.

Definition 2.3. An LTI system or model with impulse response $g(k)$ is stable if $\sum_{k=-\infty}^{\infty}|g(k)|<+\infty$.

Here, we will only study stable and causal output error models. Hence, the mean-square error optimal LTI approximation of a certain nonlinear system is simply the stable and causal LTI model $G_{0, O E}$ that minimizes

$$
\mathrm{E}\left((y(t)-G(q) u(t))^{2}\right) .
$$

This model is often called the Wiener filter for prediction of $y(t)$ from $(u(t-$ $k))_{k=0}^{\infty}$ (Wiener, 1949). However, we will instead call $G_{0, O E}$ the Output Error LTI Second Order Equivalent (OE-LTI-SOE) of the nonlinear system.

There are two main reasons for the change of name from the commonly used Wiener filter to OE-LTI-SOE. First, we want to avoid any ambiguities. Many different Wiener filters can be constructed for a given pair of input and output signals. Here, however, we are only interested in the Wiener filter that predicts $y(t)$ from $(u(t-k))_{k=0}^{\infty}$.

The second reason for the change of name is that we want to emphasize that the OE-LTI-SOE is an equivalent to the nonlinear system in the sense that it can explain the causal part of the cross-covariance function $R_{y u}(\tau)$ between the input and output of the system. This observation, which is rather obvious for OE-LTI-SOEs (see Corollary 2.2), becomes more interesting if LTI models that contain a general error description, i.e., models with $H \neq 1$, are studied. LTISOEs can be defined also in this case. It turns out that these LTI equivalents can explain both the covariance function $R_{y}(\tau)$ and the cross-covariance function $R_{y u}(\tau)$. Hence, such models are equivalents to the nonlinear system when it comes to second order properties.

It should be noted that we are not only interested in the filtering and prediction capabilities of the OE-LTI-SOE, but also in the model itself. For example, 
we are not only interested in how good an estimate of $y(t)$ the model can produce, but also in issues like how the model order and model coefficients depend on the nonlinear system and on the input signal. The notion of an OE-LTI-SOE of a nonlinear system is summarized in the following definition.

Definition 2.4. Consider a nonlinear system with input $u(t)$ and output $y(t)$ such that Assumptions A1 and A2 are fulfilled. The Output Error LTI Second Order Equivalent (OE-LTI-SOE) of this system is the stable and causal LTI model $G_{0, O E}(q)$ that minimizes the mean-square error $\mathrm{E}\left((y(t)-G(q) u(t))^{2}\right)$, i.e.,

$$
G_{0, O E}(q)=\underset{G \in \mathcal{G}}{\arg \min } \mathrm{E}\left((y(t)-G(q) u(t))^{2}\right),
$$

where $\mathcal{G}$ denotes the set of all stable and causal LTI models.

The concept of LTI-SOEs has been discussed, for example, in Ljung (2001) and Enqvist (2003). Some of the material of this paper is based on Enqvist and Ljung (2003). Some intriguing examples of OE-LTI-SOEs based on the theory presented here are given in Enqvist and Ljung (2004). It should immediately be pointed out that the OE-LTI-SOE of a nonlinear system depends on which input signal that is used. Hence, we can only talk about the OE-LTI-SOE of a nonlinear system with respect to a particular input signal. The following theorem is a direct consequence of classic Wiener filter theory.

\section{Theorem 2.1}

Consider a nonlinear system with input $u(t)$ and output $y(t)$ such that Assumptions $A 1$ and $A 2$ are fulfilled. Then the OE-LTI-SOE $G_{0, O E}$ of this system is

$$
G_{0, O E}(z)=\frac{1}{r_{u} L(z)}\left[\frac{\Phi_{y u}(z)}{L\left(z^{-1}\right)}\right]_{\text {causal }},
$$

where $[\ldots]_{\text {causal }}$ denotes taking the causal part and where $L(z)$ is the canonical spectral factor of $\Phi_{u}(z)$ from (3).

Proof: See, for example, Ljung (1999, p. 276) or Kailath et al. (2000, pp. 231233).

In general, the OE-LTI-SOE has to be calculated as in (4), which means that the canonical spectral factor $L(z)$ of the input z-spectrum has to be obtained. However, in some cases this is not necessary and the OE-LTI-SOE can be calculated using a simplified expression. This is shown in the following corollary.

\section{Corollary 2.1}

Consider a nonlinear system with input $u(t)$ and output $y(t)$ such that Assumptions A1 and A2 are fulfilled, and assume that the ratio $\Phi_{y u}(z) / \Phi_{u}(z)$ defines a stable and causal LTI model. Then

$$
G_{0, O E}(z)=\frac{\Phi_{y u}(z)}{\Phi_{u}(z)} .
$$

Proof: Assume that

$$
C(z)=\frac{\Phi_{y u}(z)}{\Phi_{u}(z)}
$$


is a stable and causal transfer function. Then

$$
\Phi_{y u}(z)=C(z) \Phi_{u}(z)=C(z) L(z) r_{u} L\left(z^{-1}\right)
$$

and (4) gives

$$
G_{0, O E}(z)=\frac{1}{r_{u} L(z)}\left[\frac{C(z) L(z) r_{u} L\left(z^{-1}\right)}{L\left(z^{-1}\right)}\right]_{\text {causal }}=C(z),
$$

since $C(z) L(z) r_{u}$ is a stable and causal transfer function.

The following corollary shows that the OE-LTI-SOE can explain the causal part of $\Phi_{y u}(z)$.

\section{Corollary 2.2}

Consider a nonlinear system with input $u(t)$ and output $y(t)$ such that Assumptions $A 1$ and $A 2$ are fulfilled. Let the residuals be defined by

$$
\eta_{0}(t)=y(t)-G_{0, O E}(q) u(t) .
$$

Then

$$
\Phi_{\eta_{0} u}(z)=\Phi_{y u}(z)-G_{0, O E}(z) \Phi_{u}(z)
$$

is strictly anticausal.

Proof: The requirement that $G_{0, O E}$ should minimize $\mathrm{E}\left((y(t)-G(q) u(t))^{2}\right)$ is equivalent to the Wiener-Hopf condition

$$
R_{y u}(\tau)-\sum_{k=0}^{\infty} g_{0, O E}(k) R_{u}(\tau-k)=0, \quad \tau \geq 0 .
$$

The result follows directly from (7).

For most systems, the order of the OE-LTI-SOE is unknown. In practice, this implies that several output error models have to be estimated and that a validation procedure has to be used in order to find the best model. Naturally, there is no guarantee that the correct order of the OE-LTI-SOE will be found. As a matter of fact, the OE-LTI-SOE can sometimes be an infinite order model. Hence, it is interesting to characterize in what sense an output error model with lower order than the OE-LTI-SOE approximates the OE-LTI-SOE.

This is a relevant question also when the true system is an LTI system. In that case, it can be shown that a low order model will approximate the true system mainly for frequencies where $\Phi_{u}\left(e^{i \omega}\right)$ is large (Ljung, 1999, p. 266). As a matter of fact, this result holds also when the true system is nonlinear. In this case, a low order output error model will approximate the OE-LTI-SOE instead of the true system. This approximation will be as good as possible for frequencies where $\Phi_{u}\left(e^{i \omega}\right)$ is large according to the following theorem. This theorem is basically a special case of Theorem 4.1 in Ljung (2001) and the proof is very similar to the outlined proof in Problem 8G.5 in Ljung (1999).

\section{Theorem 2.2}

Consider a nonlinear system with input $u(t)$ and output $y(t)$ such that Assumptions $A 1$ and $A 2$ are fulfilled. Let $G_{0, O E}$ be the corresponding OE-LTI-SOE 
according to Theorem 2.1. Suppose that a parameterized stable and causal output error model $G(q, \theta)$ is fitted to the signals $u$ and $y$ according to

$$
\hat{\theta}=\underset{\theta}{\arg \min } \mathrm{E}\left(\eta(t, \theta)^{2}\right),
$$

where

$$
\eta(t, \theta)=y(t)-G(q, \theta) u(t) .
$$

Then it follows that

$$
\hat{\theta}=\underset{\theta}{\arg \min } \int_{-\pi}^{\pi}\left|G_{0, O E}\left(e^{i \omega}\right)-G\left(e^{i \omega}, \theta\right)\right|^{2} \Phi_{u}\left(e^{i \omega}\right) d \omega .
$$

Proof: See Appendix A.

Theorem 2.2 shows that a low order output error model approximation of an OE-LTI-SOE results in the same kind of approximation as a low order approximation of an LTI system. More specifically, (10) shows that if $\Phi_{u}\left(e^{i \omega}\right)$ is large in a certain frequency region, the parameter vector $\theta$ will be chosen such that

$$
\left|G_{0, O E}\left(e^{i \omega}\right)-G\left(e^{i \omega}, \theta\right)\right|
$$

is small in that frequency region.

However, it is important to remember that there is a major difference between the linear and the nonlinear cases. If the true system is an LTI system, it is always desirable to approximate it as well as possible, at least for some frequencies. On the other hand, if the system is nonlinear, there is no guarantee that the OE-LTI-SOE is a good model of the system for any other input signals than the one it was defined for. Actually, it might be a bad model also for this signal. For example, if a second order output error model is estimated and the input power is focused in a certain frequency region, the model will in general approximate a different OE-LTI-SOE than if, for example, a white input signal had been used.

These observations make it much harder to design the input such that it is suitable for low order LTI approximations when the system is nonlinear. Some examples of input signals that might be suitable for this purpose will be given later in this paper.

So far, we have made no explicit assumptions about the structure of the nonlinear system. Although structural assumptions are not necessary for the existence of the OE-LTI-SOE, it is hard to draw any conclusions about the properties and usefulness of these second order equivalents without any further information about the nonlinear system.

One important structural property of a system is how the noise enters. For the results in this paper, we will need the following assumption that says that the noise is additive and uncorrelated with the input and the noise-free output.

Assumption A3. Assume that the output $y(t)$ can be written

$$
y(t)=y_{n f}(t)+w(t),
$$

where $y_{n f}$ is the noise-free response of the nonlinear system and not dependent on other external signals than $u$, and where $w$ is a noise term which is uncorrelated with $u$ and $y_{n f}$ and which has zero mean. 
In addition to the assumption of additive noise, we will here assume that the system is a nonlinear finite impulse response (NFIR) system, i.e., a system that can be written

$$
y(t)=f\left((u(t-k))_{k=0}^{M}\right)+w(t)
$$

for some $M \in \mathbb{N}$. Here, the compact notation

$$
f\left((u(t-k))_{k=0}^{M}\right)
$$

simply means $f(u(t), u(t-1), \ldots, u(t-M))$. Intuitively, the natural LTI approximation of an NFIR system is an FIR model. However, the mean-square error optimal LTI approximation, i.e., the OE-LTI-SOE, of such a system will in general be an LTI system with an infinite impulse response.

This might not be a problem if the impulse response length $M$ of the NFIR system is known, since it is always possible to estimate an FIR model with the same impulse response length in that case. Although this model might not be the optimal LTI model, it will at least have a structure that probably can be viewed as reasonable compared to the structure of the nonlinear system.

However, in the more realistic case that $M$ is unknown, the structure of the OE-LTI-SOE becomes important. If an NFIR system with impulse response length $M$ has an OE-LTI-SOE which is an FIR model with impulse response length $M$, it will be rather easy to find an appropriate linear FIR model of this system. When the number of measurements tends to infinity, the parameters of a chosen FIR model will converge to the parameter values given by Theorem 2.2. The problem of finding the impulse response length $M$ of the NFIR system can thus be solved by estimating linear FIR models with different impulse response lengths. If too large an impulse response length is chosen in the model, the parameters that correspond to the extra terms in the impulse response will simply approach zero asymptotically, just as if the NFIR system would have been a linear FIR system. Hence, it is possible to estimate $M$ without more effort than if the true system would have been linear.

On the other hand, if an NFIR system with impulse response length $M$ has an OE-LTI-SOE with an infinite impulse response length, it will be impossible to estimate $M$ using only linear approximations. In this case, an increase of the impulse response length in an estimated FIR model will reduce the variance of the model residuals and make the model a better approximation of the OELTI-SOE according to Theorem 2.2. However, since the OE-LTI-SOE has an infinite impulse response, no information about $M$ can be derived from the FIR approximations of it.

With the previous discussion in mind, it seems that it often should be desirable to preserve the finite impulse response property when an NFIR system is approximated by its OE-LTI-SOE. In the next section, we will present a necessary and sufficient condition on the input signal for the OE-LTI-SOE of an arbitrary NFIR system to be an FIR model. It will be shown that this condition is that the input process should be separable of a certain order (in Nuttall's sense (Nuttall, 1958)). 


\section{OE-LTI-SOEs of NFIR Systems with Separa- ble Input Processes}

We will here consider NFIR systems (12) with input signals $u(t)$ that fulfill the conditions in Assumption A1, i.e., real-valued inputs with zero mean, an exponentially bounded covariance function and a z-spectrum with a canonical spectral factorization. For each choice of such a stochastic process $u$, let $D_{u}$ be a class of Lebesgue integrable functions such that

$$
\begin{aligned}
D_{u}=\{ & f: \mathbb{R}^{M+1} \rightarrow \mathbb{R}: \mathrm{E}\left(f\left((u(t-k))_{k=0}^{M}\right)\right)=0, \\
& \mathrm{E}\left(f\left((u(t-k))_{k=0}^{M}\right)^{2}\right)<\infty \\
& \left.R_{y u}(\sigma)=\mathrm{E}\left(f\left((u(t-k))_{k=0}^{M}\right) u(t-\sigma)\right) \text { exist } \forall \sigma \in \mathbb{Z}\right\} .
\end{aligned}
$$

Note that the conditions in the definition of the class of functions $D_{u}$ are weaker than the related conditions on the output signal in Assumption A2. Here, we will use the following notation:

$$
\begin{aligned}
\mathbf{R}_{U} & =\left(\begin{array}{cccc}
R_{u}(0) & R_{u}(1) & \ldots & R_{u}(M) \\
R_{u}(1) & R_{u}(0) & \ldots & R_{u}(M-1) \\
\vdots & & & \vdots \\
R_{u}(M) & R_{u}(M-1) & \ldots & R_{u}(0)
\end{array}\right) \\
\mathbf{R}_{Y U} & =\left(\begin{array}{llll}
R_{y u}(0) & R_{y u}(1) & \ldots & \left.R_{y u}(M)\right)^{T}
\end{array}\right.
\end{aligned}
$$

We will in this section discuss under which conditions the OE-LTI-SOE of an NFIR system will be an FIR model. In this discussion, we will need the notion of the mean-square error optimal FIR model of a system. The following lemma is a classic result (see, for example, Kailath et al., 2000, Theorems 3.2.1 and 3.2.2) and holds for each fixed choice of $u$.

\section{Lemma 3.1 (FIR approximation)}

Consider an input signal $u$ that fulfills the conditions in Assumption A1 and for which $\mathbf{R}_{U}>0$. Then for each NFIR system $f$ in the corresponding class $D_{u}$, there exists a unique linear FIR model of length $M$

$$
G_{0, F I R}(z)=\sum_{k=0}^{M} \bar{b}_{f}(k) z^{-k}
$$

that is an optimal FIR(M) approximation in the mean-square error sense. This FIR model has parameters

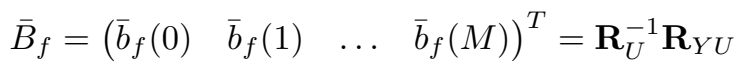

and satisfies

$$
R_{y u}(\sigma)=\sum_{k=0}^{M} \bar{b}_{f}(k) R_{u}(\sigma-k), \quad \sigma=0,1, \ldots, M .
$$

Proof: See, for example, Kailath et al. (2000). 
From (15) we see that $G_{0, F I R}$ can explain the cross-covariance function $R_{y u}(\sigma)$ for $\sigma=0,1, \ldots, M$. However, sometimes it can actually explain the complete cross-covariance function, i.e.,

$$
R_{y u}(\sigma)=\sum_{k=0}^{M} \bar{b}_{f}(k) R_{u}(\sigma-k), \quad \forall \sigma \in \mathbb{Z}
$$

or, equivalently,

$$
\Phi_{y u}(z)=G_{0, F I R}(z) \Phi_{u}(z) .
$$

In this case, we know from Corollary 2.1 that $G_{0, F I R}$ is not only the mean-square error optimal FIR(M) approximation of the system, but also the OE-LTI-SOE of the system. It turns out that this will always be true if the input process is separable of order $M+1$. Separability of a process means that certain conditional expectations are linear. This is stated more clearly in the following definition.

Definition 3.1 (Separability of order $M+1$ ). Consider an integer $M \geq 0$ and a stationary stochastic process $u$ with zero mean. This process is separable of order $M+1$ if

$$
\begin{aligned}
& \mathrm{E}(u(t-\sigma) \mid u(t), u(t-1) \ldots, u(t-M)) \\
& =\sum_{i=0}^{M} a_{\sigma, i} u(t-i), \quad \forall \sigma \in \mathbb{Z},
\end{aligned}
$$

i.e., the conditional expectation is linear in $u$.

In Nuttall (1958), the notion of separability of order one is discussed in detail and it is also mentioned briefly (on p. 76) that this notion might be extended to separability of higher orders by considering integrals like

$$
\int_{-\infty}^{\infty} x_{t} p\left(x_{t}, x_{t-\tau_{1}}, x_{t-\tau_{2}}\right) d x_{t} .
$$

However, no further conclusions are drawn in Nuttall (1958) and to the authors' knowledge, no such extension has been made elsewhere.

Since (17) is a well-known property of Gaussian signals (see, for example, Brockwell and Davis, 1987, p. 64), it immediately follows that such signals are separable of order $M+1$ for any $M \in \mathbb{N}$. Furthermore, it is easy to see that white, possibly non-Gaussian, signals fulfill (17) too. A nontrivial example of a separable process is described in the following example.

\section{Example 3.1}

Consider a process $u$ defined as

$$
u(t)=e(t)+e(t-1),
$$

where $e$ is a white process with exponential distribution over the interval $[-1$, $+\infty)$ such that $\mathrm{E}(e(t))=0$ and $\mathrm{E}\left(e(t)^{2}\right)=1$. These properties follow if each random variable $e(t)$ has the probability density function $p(x)=e^{-(x+1)}$ for $x \geq-1$. 
Since the process $e$ is white, $u(t+\tau)$ and $u(t)$ are independent if $|\tau|>1$. Hence, $\mathrm{E}(u(t+\tau) \mid u(t))=0$ for $|\tau|>1$. Furthermore, we have that

$$
\begin{aligned}
\mathrm{E}(u(t+1) \mid u(t))= & \underbrace{\mathrm{E}(e(t+1) \mid e(t)+e(t-1))}_{=0} \\
& +\mathrm{E}(e(t) \mid e(t)+e(t-1)) \\
= & \mathrm{E}(e(t) \mid e(t)+e(t-1)), \\
\mathrm{E}(u(t-1) \mid u(t))= & \mathrm{E}(e(t-1) \mid e(t)+e(t-1)) \\
& +\underbrace{\mathrm{E}(e(t-2) \mid e(t)+e(t-1))}_{=0} \\
= & \mathrm{E}(e(t-1) \mid e(t)+e(t-1)) .
\end{aligned}
$$

From these expressions we see that $u$ is separable of order one if $e$ is such that

$$
\begin{aligned}
& \mathrm{E}(e(t) \mid e(t)+e(t-1)=c) \\
& =\mathrm{E}(e(t-1) \mid e(t)+e(t-1)=c)=b \cdot c
\end{aligned}
$$

for some constant $b$ that does not depend on $c$. We will now show that these equalities hold.

Let $X$ and $Y$ be two independent random variables with probability density functions

$$
p_{X}(x)= \begin{cases}e^{-(x+1)} & \text { if } x \geq-1 \\ 0 & \text { if } x<-1\end{cases}
$$

and

$$
p_{Y}(y)= \begin{cases}e^{-(y+1)} & \text { if } y \geq-1 \\ 0 & \text { if } y<-1\end{cases}
$$

and let $W=X+Y$. Then the joint probability density function for $X$ and $W$ is

$$
\begin{aligned}
& p_{X, W}(x, w)=p_{X}(x) p_{Y}(w-x) \\
& = \begin{cases}e^{-(w+2)} & \text { if }-1 \leq x \leq w+1 \\
0 & \text { otherwise }\end{cases}
\end{aligned}
$$

For $w \geq-2$, it follows that

$$
\begin{aligned}
p_{W}(w) & =\int_{-1}^{w+1} p_{X, W}(x, w) d x \\
& =\int_{-1}^{w+1} e^{-(w+2)} d x=(w+2) e^{-(w+2)}
\end{aligned}
$$

such that

$$
p_{W}(w)= \begin{cases}(w+2) e^{-(w+2)} & \text { if } w \geq-2 \\ 0 & \text { if } w<-2\end{cases}
$$

This gives

$$
p_{X \mid W=c}(x)= \begin{cases}\frac{p_{X, W}(x, c)}{p_{W}(c)}=\frac{1}{c+2} & \text { if }-1 \leq x<c+1 \\ 0 & \text { otherwise }\end{cases}
$$


and

$$
\mathrm{E}(X \mid W=c)=\int_{-1}^{c+1} \frac{x}{c+2} d x=\frac{c}{2} .
$$

Replacing $W$ with $e(t)+e(t-1)$ and $X$ with either $e(t)$ or $e(t-1)$ in (19) shows that (18) holds with $b=1 / 2$. Hence,

$$
\mathrm{E}(u(t+1) \mid u(t)=c)=\mathrm{E}(u(t-1) \mid u(t)=c)=\frac{c}{2}
$$

and $u$ is thus separable of order one.

Besides the results about mean-square error optimal stable and causal LTI predictors, which here have been used to define OE-LTI-SOEs, classic Wiener filtering theory also contains results about mean-square error optimal stable noncausal LTI predictors, usually known as Wiener smoothers (see, for example, Kailath et al., 2000, Theorem 7.3.1). For a nonlinear system with input $u$ and output $y$ that satisfy Assumptions A1 and A2, these results show that the best, in mean-square error sense, stable but possibly noncausal LTI approximation of this system is given by the ratio

$$
\frac{\Phi_{y u}(z)}{\Phi_{u}(z)} .
$$

A simple example of a causal nonlinear system with an input such that (20) becomes noncausal can be found in Forssell and Ljung (2000). However, for a separable input, this cannot happen since the following result holds.

\section{Theorem 3.1}

Consider a fixed $M \geq 0$ and a certain input signal $u$ that fulfills the conditions in Assumption A1, and for which $\mathbf{R}_{U}>0$ and $\mathrm{E}(|u(t)|)<\infty$. Consider NFIR systems

$$
y(t)=y_{n f}(t)+w(t)=f\left((u(t-k))_{k=0}^{M}\right)+w(t),
$$

where the noise $w(t)$ is such that Assumption A3 (see (11)) is fulfilled for all $f$. Then the OE-LTI-SOE of such a system will be well-defined and equal to a linear FIR model

$$
G_{0, O E}(z)=\frac{\Phi_{y u}(z)}{\Phi_{u}(z)}=\sum_{k=0}^{M} \bar{b}_{f}(k) z^{-k}
$$

where $\bar{B}_{f}=\mathbf{R}_{U}^{-1} \mathbf{R}_{Y U}$ for all $f \in D_{u}$, if and only if $u$ is separable of order $M+1$.

Proof: See Appendix B.

Theorem 3.1 shows that separability of order $M+1$ is a necessary and sufficient condition for the OE-LTI-SOE to be equal to an FIR model of length $M$ for all NFIR systems defined by functions in $D_{u}$. Furthermore, this theorem shows that even if we consider noncausal LTI models, a separable input will give an optimal model that is a causal FIR model.

In many cases, it is possible to shed some light on a theoretical result by interpreting it in a geometrical framework. This can as a matter of fact be done 
also in our case. For a fixed $t$, we can view the output $y(t)$ and the components of the input signal $u(\tau), \tau \in \mathbb{Z}$ as vectors in an infinite dimensional inner-product space with the inner product $\langle u, v\rangle=\mathrm{E}(u v)$ (see Brockwell and Davis, 1987).

The output from the OE-LTI-SOE of the NFIR system will in this framework be the orthogonal projection of $y(t)$ into the linear subspace that is spanned by $u(t), u(t-1), \ldots, u(t-\infty)$. From $(21)$ we can draw the conclusion that this projection actually lies in the finite dimensional linear subspace that is spanned by $u(t), u(t-1), \ldots, u(t-M)$ if $u$ is separable.

As mentioned above, the set of all Gaussian signals is a subset of the set of separable signals. Hence, for an NFIR system with a Gaussian input, the cross-covariance function between $y$ and $u$ can always be written as in (16). This result is a kind of generalization of Bussgang's theorem (Theorem 1.1) to NFIR systems. The reason why (16) is not a proper generalization of Bussgang's theorem is that it is not obvious that the coefficients $\bar{b}_{f}(k)$ can be calculated as expectations of derivatives of $f$ in the same way as $b_{0}=\mathrm{E}\left(f^{\prime}(u(t))\right)$ in Theorem 1.1. However, using a direct proof based on the properties of Gaussian probability density functions, this property of the coefficients can also be shown.

\section{OE-LTI-SOEs of NFIR Systems with Gaussian Input Processes}

The generalization of Bussgang's theorem to NFIR systems can be found in, for example, Scarano et al. (1993) and has also previously been used in the research area of stochastic mechanical vibrations (see, for example, Lutes and Sarkani, 1997, Chap. 9). We will however restate the result here under the following technical assumptions.

Assumption A4: Assume that the real-valued functions $f(x)$ and $p(\tilde{x})$, where $x \in \mathbb{R}^{N}$ and $\tilde{x}=\left(x^{T}, x_{N+1}\right)^{T} \in \mathbb{R}^{N+1}$, are such that $f \cdot p, f_{x_{i}}^{\prime} \cdot p$ and $f \cdot \tilde{x}_{i} \cdot p, i=1, \ldots,(N+1)$ all belong to $L^{1}\left(\mathbb{R}^{N+1}\right)$ and that $f(x) p(\tilde{x}) \rightarrow 0$ when $|\tilde{x}| \rightarrow+\infty$. (Here, $f_{x_{i}}^{\prime}$ is the partial derivative of $f$ with respect to $\left.x_{i}\right)$.

Assumption A5: Consider two stationary stochastic processes $u$ and $y$ such that $y(t)=f\left((u(t-k))_{k=0}^{M}\right)$. Assume that $u$ is a Gaussian process with zero mean and that $\mathrm{E}(y(t))=0$. Form random vectors

$$
\omega_{\sigma}=(u(t), u(t-1), \ldots, u(t-M), u(t-\sigma))^{T}
$$

with $\sigma<0$ or $\sigma>M$. Let $P_{\sigma}$ and $p_{\sigma}$ denote the covariance matrices and joint probability density functions of these vectors, respectively. Assume that $\operatorname{det} P_{\sigma} \neq 0$ and that $f$ and $p_{\sigma}$ satisfy Assumption A4 for all $\sigma<0$ or $\sigma>M$.

Assumptions A4 and A5 assure that the input is Gaussian and that the function $f(x)$ does not grow too fast. Assumption A4 holds if, for example, $f$ is a polynomial and $p$ is a Gaussian probability density function. These assumptions are used in the following theorem. 


\section{Theorem 4.1}

Let $y(t)=f\left((u(t-k))_{k=0}^{M}\right)$ be an NFIR system with a stationary Gaussian process $u$ as input. Assume that $u$ and $y$ satisfy Assumption A5. Then it follows that

$$
R_{y u}(\tau)=\sum_{k=0}^{M} b(k) R_{u}(\tau-k), \quad \forall \tau \in \mathbb{Z},
$$

where

$$
b(k)=\mathrm{E}\left(f_{u(t-k)}^{\prime}\left((u(t-j))_{j=0}^{M}\right)\right) .
$$

Proof: See Appendix C. Scarano et al. (1993) give a proof of this result under different technical assumptions.

As mentioned above, the previous theorem can be viewed as a generalization of Bussgang's theorem to NFIR systems. Using z-transforms, the result (23) can also be written as

$$
\Phi_{y u}(z)=B(z) \Phi_{u}(z),
$$

where $B(z)=\sum_{k=0}^{M} b(k) z^{-k}$. This relation can be used to characterize the OE-LTI-SOE of an NFIR system with a Gaussian input. As has been previously mentioned, the OE-LTI-SOE is in general obtained by the Wiener filter construction in (4). However, from (24) we see that the ratio $\Phi_{y u}(z) / \Phi_{u}(z)$ is stable and causal if the nonlinear system is an NFIR system with a Gaussian input. Hence, with Corollary 2.1 in mind we can state the following theorem.

\section{Theorem 4.2}

Consider an NFIR system

$$
y(t)=f\left((u(t-k))_{k=0}^{M}\right)+w(t)
$$

with a Gaussian input $u(t)$ such that Assumptions A1, A2, A3 and A5 are satisfied. Then the OE-LTI-SOE of this system is the linear FIR model

$$
G_{0, O E}(z)=\frac{\Phi_{y u}(z)}{\Phi_{u}(z)}=\sum_{k=0}^{M} b(k) z^{-k}
$$

where

$$
b(k)=\mathrm{E}\left(f_{u(t-k)}^{\prime}\left((u(t-j))_{j=0}^{M}\right)\right) .
$$

The fact that expression (26) holds for a Gaussian input but not for a general separable input might seem like a minor difference. However, it will be shown in the next section that (26) can be rather useful if the purpose of estimating a linear model is to obtain information about the structure of the nonlinear system. In this case, a Gaussian process is a more suitable choice of input signal than a general separable process. Furthermore, Gaussianity of a process is preserved under linear filtering while separability in general is not. An application where this fact is crucial will also be described in the next section.

\section{Applications}

As mentioned above, the characterization (25) of the OE-LTI-SOE of an NFIR system with a Gaussian input is not only theoretically interesting, but can also be useful in some applications of system identification. We will here briefly discuss two such applied identification problems. 


\subsection{Structure Identification of NFIR Systems}

The most obvious application of the result (25) is perhaps to use it for guidance when an NFIR system is going to be identified. However, linear models are not useful for all types of NFIR systems. Any NFIR system can be written as a sum of an even and an odd function. Since all Gaussian probability density functions with zero mean are even functions, the OE-LTI-SOE of an NFIR system is only influenced by the odd part of the system.

Hence, we will here only consider odd NFIR systems, i.e., NFIR systems $y(t)=f\left(\left(u\left(t-n_{k}-j\right)\right)_{j=0}^{M}\right)$ where

$$
f\left(\left(-u\left(t-n_{k}-j\right)\right)_{j=0}^{M}\right)=-f\left(\left(u\left(t-n_{k}-j\right)\right)_{j=0}^{M}\right) .
$$

When such an odd NFIR system is going to be identified, it is in general not obvious how the time delay $n_{k}$ and order $M$ should be estimated in an efficient way. However, if the input is Gaussian and sufficiently many measurements can be collected, $n_{k}$ and $M$ can both be obtained from an impulse response estimate. Such an estimate can be computed very efficiently by means of the least squares method.

Furthermore, if only a few of the input terms $u\left(t-n_{k}\right), u\left(t-n_{k}-1\right), \ldots$, $u\left(t-n_{k}-M\right)$ enter the system in a nonlinear way, it might be interesting to know which these terms are. If a nonlinear model of the system is desired, this knowledge can be used to reduce the complexity of the proposed model. A coefficient $b(j)$ in $(25)$ will be invariant of the input properties if the corresponding input term $u(t-j)$ only affects the system linearly, while an input term that affects the system in a nonlinear way will have an input dependent $b$-coefficient in $(25)$.

This fact makes it possible to extract information about which nonlinear terms are present in the system simply by looking at the differences between FIR models that have been estimated with different Gaussian input signals. The coefficients that correspond to an input term that enters the system in a nonlinear way will be different in these estimates, provided that the covariance functions of the inputs are different. This idea is used in the following example.

\section{Example 5.1}

Consider the nonlinear system $y(t)=u(t)+u(t-1)^{3}$ and assume that the input to this system is Gaussian and such that the conditions in Theorem 4.2 are fulfilled. Then the OE-LTI-SOE of this system will be

$$
G_{0, O E}(q)=b(0)+b(1) q^{-1},
$$

where $b(0)=1$ and $b(1)=3 R_{u}(0)$. If the variance of the input is changed, $b(1)$ will change too, while $b(0)$ will remain equal to one. Hence, it is easy to see which input signal component affects $y(t)$ in a nonlinear way.

\subsection{Identification of Generalized Wiener-Hammerstein Systems}

In the introduction, we mentioned that Bussgang's theorem has been used to show important results concerning the identification of Hammerstein and 


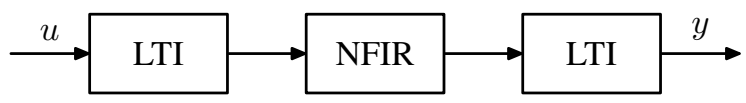

Figure 2: A generalized Wiener-Hammerstein system.

Wiener systems (see, for example, Billings and Fakhouri, 1982). In principle, these results state that an estimated LTI model will converge to a scaled version of the linear part of a Hammerstein or Wiener system when the number of measurements tends to infinity, provided that the input is Gaussian. These results simplify the identification of Wiener and Hammerstein systems significantly.

Hence, it is interesting to investigate if the result (25) about the OE-LTISOEs of NFIR systems can be used to prove similar results for extended classes of systems. In this section, we will study a type of systems that we will call generalized Wiener-Hammerstein systems.

More specifically, we will call a nonlinear system a generalized WienerHammerstein system if it consists of an LTI system $n(t)=G_{1}(q) u(t)$ followed by an NFIR system $v(t)=f\left((n(t-k))_{k=0}^{M}\right)$ followed by an LTI system $y(t)=G_{2}(q) v(t)$ as is shown in Figure 2. The following corollary to Theorem 4.2 shows that the OE-LTI-SOE of such a system has a certain structure.

\section{Corollary 5.1}

Consider a generalized Wiener-Hammerstein system $y(t)=G_{2}(q) v(t)+w(t)$ where $v(t)=f\left((n(t-k))_{k=0}^{M}\right)$ and $n(t)=G_{1}(q) u(t)$ and where $G_{1}(q)$ and $G_{2}(q)$ are stable and causal LTI systems. Assume that $u(t)$ is Gaussian and that $u(t)$ and $y(t)$ fulfill Assumptions A1, A2 and A3. Assume also that $n(t)$ and $v(t)$ fulfill Assumptions A1, A2 and A5. Then the OE-LTI-SOE of this system is

$$
G_{0, O E}(z)=G_{2}(z) B(z) G_{1}(z)
$$

where $B(z)=\sum_{k=0}^{M} b(k) z^{-k}$ and

$$
b(k)=\mathrm{E}\left(f_{n(t-k)}^{\prime}\left((n(t-j))_{j=0}^{M}\right)\right) .
$$

Proof: We have

$$
\begin{aligned}
\Phi_{y u}(z) & =G_{2}(z) \Phi_{v u}(z), \\
\Phi_{v n}(z) & =\Phi_{v u}(z) G_{1}\left(z^{-1}\right), \\
\Phi_{n}(z) & =G_{1}(z) \Phi_{u}(z) G_{1}\left(z^{-1}\right) .
\end{aligned}
$$

In addition, Theorem 4.2 gives that

$$
\Phi_{v n}(z)=B(z) \Phi_{n}(z) .
$$

Inserting (28b) and (28c) in (29) gives

$$
\Phi_{v u}(z)=B(z) G_{1}(z) \Phi_{u}(z),
$$

and inserting (28a) in (30) gives

$$
\Phi_{y u}(z)=G_{2}(z) B(z) G_{1}(z) \Phi_{u}(z) .
$$

Hence, (27) follows from Corollary 2.1. 
Corollary 5.1 shows that the OE-LTI-SOE of a generalized Wiener-Hammerstein system with a Gaussian input will be $G_{2}(z) B(z) G_{1}(z)$, and hence an estimated output error model will approach this model when the number of measurements tends to infinity. In particular, as $B(z)$ is an FIR model, this shows that the denominator of the estimated model will approach the product of the denominators of $G_{1}$ and $G_{2}$ if the degree of the model denominator polynomial is correct.

We will thus get consistent estimates of the poles of $G_{1}$ and $G_{2}$ despite the presence of the NFIR system. This is particularly useful if either $G_{1}$ or $G_{2}$ is equal to one, i.e., if we have either a generalized Hammerstein or a generalized Wiener system. The consistency of the pole estimates for a generalized Hammerstein system is verified numerically in Example 5.2.

\section{Example 5.2}

Consider a generalized Hammerstein system

$$
y(t)=G(q) f(u(t), u(t-1))+w(t),
$$

where

$$
\begin{aligned}
G(q) & =\frac{1}{1+0.6 q^{-1}+0.1 q^{-2}}, \\
f(u(t), u(t-1)) & =\arctan (u(t)) \cdot u(t-1)^{2}
\end{aligned}
$$

and where $w(t)$ is white Gaussian noise with $\mathrm{E}(w(t))=0$ and $\mathrm{E}\left(w(t)^{2}\right)=1$.

Let the input $u(t)$ be generated by linear filtering of a white Gaussian process $e(t)$ with $\mathrm{E}(e(t))=0$ and $\mathrm{E}\left(e(t)^{2}\right)=1$ such that

$$
u(t)=\frac{1-0.8 q^{-1}+0.1 q^{-2}}{1-0.2 q^{-1}} e(t)
$$

and assume that $e(t)$ and $w(s)$ are independent for all $t, s \in \mathbb{Z}$.

This input signal has been used in an identification experiment where a data set consisting of 100000 measurements of $u(t)$ and $y(t)$ was collected. The large number of measurements has been chosen since the convergence towards the OELTI-SOE might be slow. A linear output error model $\hat{G}_{O E}$ with $n_{b}=n_{f}=2$ and $n_{k}=0$ has been estimated from this data set and the result was

$$
\hat{G}_{O E}(q)=\frac{0.762-0.682 q^{-1}}{1+0.613 q^{-1}+0.102 q^{-2}} .
$$

As can easily be seen from (31), the denominator of $\hat{G}_{O E}(q)$ is indeed close to the denominator of $G(q)$. This is exactly what one would expect as the previous theoretical discussion give that the OE-LTI-SOE of the generalized Hammerstein system is the product between $G(q)$ and an FIR model $B(q)$.

The following example verifies Corollary 5.1 also for a particular generalized Wiener system. 


\section{Example 5.3}

Consider a generalized Wiener system consisting of the same linear and nonlinear blocks as the generalized Hammerstein system in Example 5.2 but with the linear block before the nonlinear, i.e.,

$$
\begin{aligned}
& y(t)=f(n(t), n(t-1))+w(t), \\
& n(t)=G(q) u(t),
\end{aligned}
$$

where

$$
\begin{aligned}
G(q) & =\frac{1}{1+0.6 q^{-1}+0.1 q^{-2}}, \\
f(n(t), n(t-1)) & =\arctan (n(t)) \cdot n(t-1)^{2},
\end{aligned}
$$

and where $w(t)$ is white Gaussian noise with $\mathrm{E}(w(t))=0$ and $\mathrm{E}\left(w(t)^{2}\right)=1$.

Let the input $u(t)$ be generated in the same way as in Example 5.2, i.e.,

$$
u(t)=\frac{1-0.8 q^{-1}+0.1 q^{-2}}{1-0.2 q^{-1}} e(t),
$$

where $e(t)$ is a white Gaussian process with $\mathrm{E}(e(t))=0$ and $\mathrm{E}\left(e(t)^{2}\right)=1$ such that $e(t)$ and $w(s)$ are independent for all $t, s \in \mathbb{Z}$.

An identification experiment has been performed on this generalized Wiener system with a realization of this $u(t)$ as input and 100000 measurements of $u(t)$ and $y(t)$ have been collected. A linear output error model $\hat{G}_{O E}(q)$ with $n_{b}=n_{f}=2$ and $n_{k}=0$ has been estimated from the measurements and the result was

$$
\hat{G}_{O E}(q)=\frac{0.929-2.053 q^{-1}}{1+0.596 q^{-1}+0.0971 q^{-2}} .
$$

From (32) we can see that the denominator of $\hat{G}_{O E}(q)$ is close to the denominator of $G(q)$ also when the data has been generated by a generalized Wiener system.

\section{Conclusions}

In this paper, we have shown that a necessary and sufficient criterion on the input signal for the OE-LTI-SOE of an arbitrary NFIR system to be an FIR model is that the input is separable of a certain order. We have also noted that the set of Gaussian processes is a subset of the set of separable processes. For Gaussian inputs, the fact that the OE-LTI-SOE of an NFIR system is an FIR model follows from a generalized version of Bussgang's theorem. Here, we have presented some applications of this result for structure identification and identification of generalized Wiener-Hammerstein systems.

\section{Acknowledgements}

The authors would like to thank the reviewers for providing several helpful suggestions. This work has been supported by the Swedish Research Council, which is hereby gratefully acknowledged. 


\section{A Proof of Theorem 2.2}

Proof: The z-spectrum of $\eta(t, \theta)$ is

$$
\begin{aligned}
\Phi_{\eta}(z, \theta)= & \left(\begin{array}{ll}
-G(z, \theta) & 1
\end{array}\right)\left(\begin{array}{cc}
\Phi_{u}(z) & \Phi_{u y}(z) \\
\Phi_{y u}(z) & \Phi_{y}(z)
\end{array}\right)\left(\begin{array}{c}
-G\left(z^{-1}, \theta\right) \\
1
\end{array}\right) \\
= & \Phi_{y}(z)-G(z, \theta) \Phi_{u y}(z)-G\left(z^{-1}, \theta\right) \Phi_{y u}(z)+G(z, \theta) \Phi_{u}(z) G\left(z^{-1}, \theta\right) \\
= & \left(G(z, \theta)-\frac{\Phi_{y u}(z)}{\Phi_{u}(z)}\right) \Phi_{u}(z)\left(G\left(z^{-1}, \theta\right)-\frac{\Phi_{y u}\left(z^{-1}\right)}{\Phi_{u}\left(z^{-1}\right)}\right) \\
& -\frac{\Phi_{y u}(z) \Phi_{y u}\left(z^{-1}\right)}{\Phi_{u}(z)}+\Phi_{y}(z) .
\end{aligned}
$$

Let

$$
\begin{aligned}
& A_{0}=\frac{1}{2 \pi} \int_{-\pi}^{\pi}\left(\Phi_{y}\left(e^{i \omega}\right)-\frac{\left|\Phi_{y u}\left(e^{i \omega}\right)\right|^{2}}{\Phi_{u}\left(e^{i \omega}\right)}\right) d \omega \\
& B_{0}=\frac{1}{2 \pi} \int_{-\pi}^{\pi} \frac{\left|\Phi_{\eta_{0} u}\left(e^{i \omega}\right)\right|^{2}}{\Phi_{u}\left(e^{i \omega}\right)} d \omega
\end{aligned}
$$

Parseval's relation gives

$$
\begin{aligned}
\mathrm{E}\left(\eta(t, \theta)^{2}\right)= & \frac{1}{2 \pi} \int_{-\pi}^{\pi} \Phi_{\eta}\left(e^{i \omega}, \theta\right) d \omega \\
= & \frac{1}{2 \pi} \int_{-\pi}^{\pi}\left|\frac{\Phi_{y u}\left(e^{i \omega}\right)}{\Phi_{u}\left(e^{i \omega}\right)}-G\left(e^{i \omega}, \theta\right)\right|^{2} \Phi_{u}\left(e^{i \omega}\right) d \omega+A_{0} \\
= & \frac{1}{2 \pi} \int_{-\pi}^{\pi}\left|G_{0, O E}\left(e^{i \omega}\right)+\frac{\Phi_{\eta_{0} u}\left(e^{i \omega}\right)}{\Phi_{u}\left(e^{i \omega}\right)}-G\left(e^{i \omega}, \theta\right)\right|^{2} \Phi_{u}\left(e^{i \omega}\right) d \omega+A_{0} \\
= & \frac{1}{2 \pi} \int_{-\pi}^{\pi}\left|G_{0, O E}\left(e^{i \omega}\right)-G\left(e^{i \omega}, \theta\right)\right|^{2} \Phi_{u}\left(e^{i \omega}\right) d \omega \\
& +\frac{1}{2 \pi} \int_{-\pi}^{\pi} \Phi_{\eta_{0} u}\left(e^{i \omega}\right)\left(G_{0, O E}\left(e^{-i \omega}\right)-G\left(e^{-i \omega}, \theta\right)\right) d \omega \\
& +\frac{1}{2 \pi} \int_{-\pi}^{\pi} \Phi_{\eta_{0} u}\left(e^{-i \omega}\right)\left(G_{0, O E}\left(e^{i \omega}\right)-G\left(e^{i \omega}, \theta\right)\right) d \omega+A_{0}+B_{0},
\end{aligned}
$$

where we have used (6) in the third equality. Since $\Phi_{\eta_{0} u}(z)$ by Corollary 2.2 is strictly anticausal and since $G_{0, O E}(z)$ and $G(z, \theta)$ both are causal, a term-byterm integration shows that

$$
\begin{aligned}
& \frac{1}{2 \pi} \int_{-\pi}^{\pi} \Phi_{\eta_{0} u}\left(e^{i \omega}\right)\left(G_{0, O E}\left(e^{-i \omega}\right)-G\left(e^{-i \omega}, \theta\right)\right) d \omega=0, \\
& \frac{1}{2 \pi} \int_{-\pi}^{\pi} \Phi_{\eta_{0} u}\left(e^{-i \omega}\right)\left(G_{0, O E}\left(e^{i \omega}\right)-G\left(e^{i \omega}, \theta\right)\right) d \omega=0 .
\end{aligned}
$$

Thus

$$
\mathrm{E}\left(\eta(t, \theta)^{2}\right)=\frac{1}{2 \pi} \int_{-\pi}^{\pi}\left|G_{0, O E}\left(e^{i \omega}\right)-G\left(e^{i \omega}, \theta\right)\right|^{2} \Phi_{u}\left(e^{i \omega}\right) d \omega+A_{0}+B_{0}
$$

and (10) follows as $A_{0}$ and $B_{0}$ are independent of $\theta$. 


\section{B Proof of Theorem 3.1}

First, we will here consider noise-free NFIR systems, i.e., nonlinear systems with impulse response lengths $M \geq 0$ that can be written as

$$
y(t)=f\left((u(t-k))_{k=0}^{M}\right) .
$$

We will use the following notation

$$
\mathbf{R}_{U, \sigma}=\left(\begin{array}{llll}
R_{u}(\sigma) & R_{u}(\sigma-1) & \ldots & R_{u}(\sigma-M)
\end{array}\right)^{T}
$$

and we will assume that $\mathbf{R}_{U}$ (see (13)) is a positive definite matrix $\left(\mathbf{R}_{U}>0\right.$ ) such that the vector

$$
C_{\sigma}=\left(\begin{array}{llll}
c_{\sigma, 0} & c_{\sigma, 1} & \ldots & c_{\sigma, M}
\end{array}\right)^{T}=\mathbf{R}_{U}^{-1} \mathbf{R}_{U, \sigma}
$$

is well-defined.

We will now show that the definition of separability implies that $a_{\sigma, i}=c_{\sigma, i}$. For $k=0,1, \ldots, M$, Definition 3.1 gives

$$
\begin{aligned}
R_{u}(\sigma-k) & =\mathrm{E}(u(t-k) u(t-\sigma)) \\
& =\mathrm{E}(\mathrm{E}(u(t-k) u(t-\sigma) \mid u(t), u(t-1) \ldots, u(t-M))) \\
& =\mathrm{E}(u(t-k) \mathrm{E}(u(t-\sigma) \mid u(t), u(t-1) \ldots, u(t-M))) \\
& =\sum_{i=0}^{M} a_{\sigma, i} \mathrm{E}(u(t-k) u(t-i))=\sum_{i=0}^{M} a_{\sigma, i} R_{u}(k-i) .
\end{aligned}
$$

Here, we have used the facts that

$$
\begin{aligned}
\mathrm{E}(Y) & =\mathrm{E}(\mathrm{E}(Y \mid X)), \\
\mathrm{E}(g(X) Y \mid X) & =g(X) \mathrm{E}(Y \mid X)
\end{aligned}
$$

(see, for example, Gut, 1995, Chap. 2). If $A_{\sigma}$ is defined as

$$
A_{\sigma}=\left(\begin{array}{llll}
a_{\sigma, 0} & a_{\sigma, 1} & \ldots & a_{\sigma, M}
\end{array}\right)^{T},
$$

the previous expression can also be written as

$$
\mathbf{R}_{U} A_{\sigma}=\mathbf{R}_{U, \sigma}
$$

This shows that $A_{\sigma}=\mathbf{R}_{U}^{-1} \mathbf{R}_{U, \sigma}=C_{\sigma}$. Hence, separability of order $M+1$ means that the property

$$
\mathrm{E}(u(t-\sigma) \mid u(t), u(t-1) \ldots, u(t-M))=\sum_{i=0}^{M} c_{\sigma, i} u(t-i), \quad \forall \sigma \in \mathbb{Z}
$$

holds.

In the next lemma, we will show that separability of $u$ is a necessary and sufficient condition for the equality (16) to hold for all $\sigma \in \mathbb{Z}$ and for all $f \in D_{u}$. 


\section{Lemma B.1 (Separability of order $M+1$ )}

Consider a fixed $M \geq 0$ and a certain choice of input signal $u$ that fulfills the conditions in Assumption A1, and for which $\mathbf{R}_{U}>0$ and $\mathrm{E}(|u(t)|)<\infty$. Let $\bar{B}_{f}$ denote the parameters of the mean-square error optimal FIR(M) approximation of each $f \in D_{u}$, i.e., $\bar{B}_{f}=\mathbf{R}_{U}^{-1} \mathbf{R}_{Y U}$ according to Lemma 3.1. Then

$$
R_{y u}(\sigma)=\sum_{k=0}^{M} \bar{b}_{f}(k) R_{u}(\sigma-k), \quad \forall \sigma \in \mathbb{Z} \text { and } \forall f \in D_{u}
$$

if and only if $u$ is separable of order $M+1$.

Proof: Using (33) and (14), it follows that

$$
\sum_{k=0}^{M} \bar{b}_{f}(k) R_{u}(\sigma-k)=\bar{B}_{f}^{T} \mathbf{R}_{U, \sigma}=\mathbf{R}_{Y U}^{T} C_{\sigma}=\sum_{i=0}^{M} c_{\sigma, i} R_{y u}(i) .
$$

IF: Assume that $u$ is separable of order $M+1$, i.e., that (35) holds. By the construction of $\bar{B}_{f}$, the equality (36) already holds for $\sigma=0,1, \ldots, M$ for all $f \in D_{u}$ (cf. (14)). Take an arbitrary $f \in D_{u}$ and let $y(t)=f\left((u(t-k))_{k=0}^{M}\right)$. Furthermore, take an arbitrary $\sigma>M$ or $\sigma<0$. Then it follows that

$$
\begin{aligned}
R_{y u}(\sigma) & =\mathrm{E}(y(t) u(t-\sigma))=\mathrm{E}(\mathrm{E}(y(t) u(t-\sigma) \mid u(t), u(t-1), \ldots, u(t-M))) \\
& =\mathrm{E}(y(t) \mathrm{E}(u(t-\sigma) \mid u(t), u(t-1), \ldots, u(t-M))) \\
& =\sum_{i=0}^{M} c_{\sigma, i} \mathrm{E}(y(t) u(t-i))=\sum_{i=0}^{M} c_{\sigma, i} R_{y u}(i)=\sum_{k=0}^{M} \bar{b}_{f}(k) R_{u}(\sigma-k),
\end{aligned}
$$

where we have used (34a) in the second equality. The third equality follows from (34b) and the fact that $y(t)$ depends only on $u(t), u(t-1), \ldots, u(t-M)$ while the fourth equality follows from (35) and the last from (37). Since both $f$ and $\sigma$ were arbitrary, (36) holds for all $\sigma \in \mathbb{Z}$ and for all $f \in D_{u}$.

ONLY IF: Assume that (36) holds for a particular $u$. Take an arbitrary $\sigma>M$ or $\sigma<0$. Using (37), (36) gives the equality

$$
\begin{gathered}
\int_{\mathbb{R}^{M+1}} f\left(x_{t}, \ldots, x_{t-M}\right)\left(\int_{-\infty}^{\infty} x_{t-\sigma} p_{\sigma}\left(x_{t}, \ldots, x_{t-M}, x_{t-\sigma}\right) d x_{t-\sigma}\right. \\
\left.-\sum_{i=0}^{M} c_{\sigma, i} x_{t-i} p\left(x_{t}, \ldots, x_{t-M}\right)\right) d x_{t} \ldots d x_{t-M}=0, \quad \forall f \in D_{u},
\end{gathered}
$$

where $p$ and $p_{\sigma}$ are the joint probability density functions of $(u(t), u(t-1), \ldots$, $u(t-M))^{T}$ and $(u(t), u(t-1), \ldots, u(t-M), u(t-\sigma))^{T}$, respectively. Let

$$
\begin{aligned}
v_{\sigma}\left(x_{t}, \ldots, x_{t-M}\right)= & \int_{-\infty}^{\infty} x_{t-\sigma} p_{\sigma}\left(x_{t}, \ldots, x_{t-M}, x_{t-\sigma}\right) d x_{t-\sigma} \\
& -\sum_{i=0}^{M} c_{\sigma, i} x_{t-i} p\left(x_{t}, \ldots, x_{t-M}\right)
\end{aligned}
$$

and define a function

$$
f_{0}\left(x_{t}, \ldots, x_{t-M}\right)=\operatorname{sign}\left(v_{\sigma}\left(x_{t}, \ldots, x_{t-M}\right)\right)-\mu_{0},
$$


where

$$
\mu_{0}=\mathrm{E}\left(\operatorname{sign}\left(v_{\sigma}\left((u(t-k))_{k=0}^{M}\right)\right)\right) .
$$

Since

$$
\begin{aligned}
\mathrm{E}\left(f_{0}\left((u(t-k))_{k=0}^{M}\right)\right) & =0, \\
\mathrm{E}\left(f_{0}\left((u(t-k))_{k=0}^{M}\right)^{2}\right) & <\infty
\end{aligned}
$$

and

$$
\begin{aligned}
& \left|\mathrm{E}\left(f_{0}\left((u(t-k))_{k=0}^{M}\right) u(t-\tau)\right)\right| \\
& =|\mathrm{E}\left(\operatorname{sign}\left(v_{\sigma}\left((u(t-k))_{k=0}^{M}\right)\right) u(t-\tau)\right)-\mu_{0} \underbrace{\mathrm{E}(u(t-\tau))}_{=0}| \\
& =\left|\mathrm{E}\left(\operatorname{sign}\left(v_{\sigma}\left((u(t-k))_{k=0}^{M}\right)\right) u(t-\tau)\right)\right| \\
& \leq \mathrm{E}\left(\left|\operatorname{sign}\left(v_{\sigma}\left((u(t-k))_{k=0}^{M}\right)\right) u(t-\tau)\right|\right) \\
& \leq \mathrm{E}(|u(t-\tau)|)<\infty, \quad \forall \tau \in \mathbb{Z},
\end{aligned}
$$

it follows that $f_{0} \in D_{u}$. Hence, (38) holds for $f=f_{0}$ and this implies that

$$
\begin{aligned}
& \int_{\mathbb{R}^{M+1}}\left|v_{\sigma}\left(x_{t}, \ldots, x_{t-M}\right)\right| d x_{t} \ldots d x_{t-M} \\
& -\mu_{0} \underbrace{\mathrm{E}(u(t-\sigma))}_{=0}+\mu_{0} \sum_{i=0}^{M} c_{\sigma, i} \underbrace{\mathrm{E}(u(t-i))}_{=0}=0 \\
& \Rightarrow \int_{\mathbb{R}^{M+1}}\left|v_{\sigma}\left(x_{t}, \ldots, x_{t-M}\right)\right| d x_{t} \ldots d x_{t-M}=0 \\
& \Rightarrow v_{\sigma}\left(x_{t}, \ldots, x_{t-M}\right)=0 \quad \text { almost everywhere. }
\end{aligned}
$$

The conditional probability density function of $u(t-\sigma)$ given $u(t)=x_{t}, u(t-1)=$ $x_{t-1}, \ldots, u(t-M)=x_{t-M}$ is

$$
p_{\sigma, c}\left(x_{t-\sigma}\right)=\frac{p_{\sigma}\left(x_{t}, \ldots, x_{t-M}, x_{t-\sigma}\right)}{p\left(x_{t}, \ldots, x_{t-M}\right)}
$$

if $p\left(x_{t}, \ldots, x_{t-M}\right)>0$. Hence, the fact that

$$
v_{\sigma}\left(x_{t}, \ldots, x_{t-M}\right)=0
$$

implies that

$$
\int_{-\infty}^{\infty} x_{t-\sigma} p_{\sigma, c}\left(x_{t-\sigma}\right) d x_{t-\sigma}=\sum_{i=0}^{M} c_{\sigma, i} x_{t-i}
$$

or, equivalently, that (35) holds for the chosen $\sigma$. Since $\sigma$ was arbitrary, (35) follows and $u$ is thus separable of order $M+1$.

Lemma B.1 is an extension of the corresponding theorem about separability of order one in Nuttall (1958). Lemma B.1 together with Corollary 2.1 give the result in Theorem 3.1 . 
Proof of Theorem 3.1: Assumption A3 gives $\Phi_{y u}(z)=\Phi_{y_{n f} u}(z)$. Hence, the OE-LTI-SOE is not influenced by the noise term $w(t)$. Since the input satisfies the conditions in Lemma B.1, we have that

$$
\Phi_{y_{n f} u}(z)=\sum_{k=0}^{M} \bar{b}_{f}(k) z^{-k} \Phi_{u}(z),
$$

where $\bar{B}_{f}=\mathbf{R}_{U}^{-1} \mathbf{R}_{Y U}$ for all $f \in D_{u}$, if and only if $u$ is separable of order $M+1$. If (39) holds for all $f \in D_{u}$, the NFIR systems that correspond to these functions have outputs that satisfy Assumption A2. Hence, the OE-LTI-SOEs of these NFIR systems are well-defined and Corollary 2.1 can be applied to show that

$$
G_{0, O E}(z)=\frac{\Phi_{y_{n f} u}(z)}{\Phi_{u}(z)}=\sum_{k=0}^{M} \bar{b}_{f}(k) z^{-k}
$$

for all $f \in D_{u}$. The theorem has thus been shown.

\section{Proof of Theorem 4.1}

First, we will prove the following lemma.

\section{Lemma C.1}

Let

$$
\tilde{x}=\left(x^{T}, x_{N+1}\right)^{T}=\left(x_{1}, x_{2}, \ldots, x_{N}, x_{N+1}\right)^{T}
$$

be a jointly Gaussian distributed random vector with zero mean and covariance matrix $C$ with $\operatorname{det} C \neq 0$. Let $f: \mathbb{R}^{N} \rightarrow \mathbb{R}$ be a differentiable function of $x$ with $\mathrm{E}(f(x))=0$ and let $p$ denote the probability density function of $\tilde{x}$. Furthermore, assume that $f$ and $p$ satisfy Assumption A4. Then

$$
\mathrm{E}(f(x) \tilde{x})=C w
$$

where

$$
w=\left(\begin{array}{c}
\mathrm{E}\left(f_{x_{1}}^{\prime}(x)\right) \\
\mathrm{E}\left(f_{x_{2}}^{\prime}(x)\right) \\
\vdots \\
\mathrm{E}\left(f_{x_{N}}^{\prime}(x)\right) \\
0
\end{array}\right) .
$$

Proof: Factorize $C$ as $C=\tilde{Q} \tilde{Q}^{T}$ and define a new stochastic vector $z$ as $z=$ $\tilde{Q}^{-1} \tilde{x}$. Then $z$ is jointly normally distributed with zero mean and a covariance matrix that is equal to the identity matrix. Let $Q$ denote the matrix that is obtained from $\tilde{Q}$ by removing the last row. Then $x=Q z$ and we get

$$
\begin{aligned}
\mathrm{E}(f(x) \tilde{x}) & =\tilde{Q} \mathrm{E}\left(f(x) \tilde{Q}^{-1} \tilde{x}\right)=\tilde{Q} \mathrm{E}(f(Q z) z) \\
& =\tilde{Q}\left(\begin{array}{c}
\mathrm{E}\left(\frac{\partial f(Q z)}{\partial z_{1}}\right) \\
\mathrm{E}\left(\frac{\partial f(Q z)}{\partial z_{2}}\right) \\
\vdots \\
\mathrm{E}\left(\frac{\partial f(Q z)}{\partial z_{N+1}}\right)
\end{array}\right)=\tilde{Q} \tilde{Q}^{T}\left(\begin{array}{c}
\mathrm{E}\left(f_{x_{1}}^{\prime}(x)\right) \\
\mathrm{E}\left(f_{x_{2}}^{\prime}(x)\right) \\
\vdots \\
\mathrm{E}\left(f_{x_{N}}^{\prime}(x)\right) \\
0
\end{array}\right)=C w .
\end{aligned}
$$


The third equality follows from the fact that $\mathrm{E}\left(h(z) z_{i}\right)=\mathrm{E}\left(h_{z_{i}}^{\prime}(z)\right)$ when $z$ has an $N(0, I)$ distribution. This equality holds since

$$
\int_{-\infty}^{\infty} g(r) r e^{-r^{2} / 2} d r=\left[-g(r) e^{-r^{2} / 2}\right]_{r=-\infty}^{\infty}+\int_{-\infty}^{\infty} g^{\prime}(r) e^{-r^{2} / 2} d r .
$$

Furthermore, the fourth equality in the derivation above follows from the chain rule, which can be written here as

$$
\frac{\partial f(Q z)}{\partial z_{i}}=\frac{\partial f(Q z)}{\partial x_{1}} Q_{1 i}+\frac{\partial f(Q z)}{\partial x_{2}} Q_{2 i}+\ldots+\frac{\partial f(Q z)}{\partial x_{N}} Q_{N i} .
$$

Lemma C.1 is used in the following proof.

Proof of Theorem 4.1: Choose an arbitrary $\sigma<0$ or $\sigma>M$ and let

$$
x=(u(t), u(t-1), \ldots, u(t-M))^{T}
$$

and $x_{N+1}=u(t-\sigma)$ in Lemma C.1. Then Equation (41) gives

$$
\mathrm{E}\left(y(t)\left(\begin{array}{c}
u(t) \\
u(t-1) \\
\vdots \\
u(t-M) \\
u(t-\sigma)
\end{array}\right)\right)=\left(\begin{array}{ccccc}
R_{u}(0) & R_{u}(1) & \ldots & R_{u}(M) & R_{u}(\sigma) \\
R_{u}(1) & R_{u}(0) & \ldots & R_{u}(M-1) & R_{u}(\sigma-1) \\
\vdots & \vdots & \ddots & \vdots & \vdots \\
R_{u}(M) & R_{u}(M-1) & \ldots & R_{u}(0) & R_{u}(\sigma-M) \\
R_{u}(\sigma) & R_{u}(\sigma-1) & \ldots & R_{u}(\sigma-M) & R_{u}(0)
\end{array}\right) w
$$

where $w_{i+1}=\mathrm{E}\left(f_{u(t-i)}^{\prime}\left((u(t-k))_{k=0}^{M}\right)\right)$ for $i=0, \ldots, M$ and $w_{M+2}=0$. Equation (42) can be written more compactly as

$$
R_{y u}(\tau)=\sum_{k=0}^{M} b(k) R_{u}(\tau-k), \quad \tau=0,1, \ldots, M, \sigma,
$$

where $b(k)=w_{k+1}=\mathrm{E}\left(f_{u(t-k)}^{\prime}\left((u(t-j))_{j=0}^{M}\right)\right)$. As $\sigma$ was chosen arbitrarily, this relation holds for all $\tau \in \mathbb{Z}$.

\section{References}

J. F. Barrett and D. G. Lampard. An expansion for some second-order probability distributions and its application to noise problems. IRE Transactions on Information Theory, 1(1):10-15, 1955.

J. S. Bendat. Nonlinear Systems Techniques and Applications. John Wiley \& Sons, 1998.

S. A. Billings and S. Y. Fakhouri. Identification of systems containing linear dynamic and static nonlinear elements. Automatica, 18(1):15-26, 1982.

P. J. Brockwell and R. A. Davis. Time Series: Theory and Methods. Springer, New York, 1987. 
J. L. Brown. On a cross-correlation property for stationary random processes. IRE Transactions on Information Theory, 3(1):28-31, 1957.

J. J. Bussgang. Crosscorrelation functions of amplitude-distorted Gaussian signals. Technical Report 216, MIT Laboratory of Electronics, 1952.

M. Enqvist. Some results on linear models of nonlinear systems. Licentiate thesis no. 1046. Department of Electrical Engineering, Linköping University, SE-581 83 Linköping, Sweden, 2003.

M. Enqvist and L. Ljung. Linear models of nonlinear FIR systems with Gaussian inputs. In Preprints of the 13th IFAC Symposium on System Identification, pages 1910-1915, Rotterdam, The Netherlands, August 2003.

M. Enqvist and L. Ljung. LTI approximations of slightly nonlinear systems: Some intriguing examples. In Preprints of the 6th IFAC Symposium on Nonlinear Control Systems, pages 639-644, Stuttgart, Germany, September 2004.

U. Forssell and L. Ljung. A projection method for closed loop identification. IEEE Transactions on Automatic Control, 45(11):2101-2106, 2000.

A. Gut. An Intermediate Course in Probability. Springer, New York, 1995.

I. M. Horowitz. Quantitative Feedback Design Theory. QFT Publications, Boulder, Colorado, 1993.

T. Kailath, A. H. Sayed, and B. Hassibi. Linear Estimation. Prentice Hall, Upper Saddle River, New Jersey, 2000.

M. J. Korenberg. Identifying noisy cascades of linear and static nonlinear systems. In Proc. 7th IFAC Symp. on Identification and System Parameter Identification, pages 421-426, York, UK, 1985.

L. Ljung. Convergence analysis of parametric identification methods. IEEE Transactions on Automatic Control, 23(5):770-783, 1978.

L. Ljung. System Identification: Theory for the User. Prentice Hall, Upper Saddle River, New Jersey, second edition, 1999.

L. Ljung. Estimating linear time-invariant models of nonlinear time-varying systems. European Journal of Control, 7(2-3):203-219, 2001.

L. D. Lutes and S. Sarkani. Stochastic Analysis of Structural and Mechanical Vibrations. Prentice Hall, Upper Saddle River, New Jersey, 1997.

D. K. McGraw and J. F. Wagner. Elliptically symmetric distributions. IEEE Transactions on Information Theory, 14(1):110-120, 1968.

P. M. Mäkilä. Optimal approximation and model quality estimation for nonlinear systems. In Preprints of the 13th IFAC Symposium on System Identification, pages 1904-1909, Rotterdam, The Netherlands, August 2003a.

P. M. Mäkilä. Squared and absolute errors in optimal approximation of nonlinear systems. Automatica, 39(11):1865-1876, 2003b. 
P. M. Mäkilä and J. R. Partington. On linear models for nonlinear systems. Automatica, 39(1):1-13, 2003.

P. M. Mäkilä and J. R. Partington. Least-squares LTI approximation of nonlinear systems and quasistationarity analysis. Automatica, 40(7):1157-1169, 2004.

A. H. Nuttall. Theory and Application of the Separable Class of Random Processes. PhD thesis, MIT, 1958.

A. Papoulis. Probability, Random Variables and Stochastic Processes. McGraw Hill, second edition, 1984.

J. R. Partington and P. M. Mäkilä. On system gains for linear and nonlinear systems. Systems \& Control Letters, 46(2):129-136, 2002.

R. Pintelon and J. Schoukens. System Identification: A Frequency Domain Approach. IEEE Press, New Jersey, 2001.

R. Pintelon and J. Schoukens. Measurement and modelling of linear systems in the presence of nonlinear distortions. Mechanical Systems and Signal Processing, 16(5):785-801, 2002.

R. Pintelon, J. Schoukens, W. Van Moer, and Y. Rolain. Identification of linear systems in the presence of nonlinear distortions. IEEE Transactions on Instrumentation and Measurement, 50(4):855-863, 2001.

S. Sastry. Nonlinear systems - Analysis, stability and control. Springer, New York, 1999.

G. Scarano, D. Caggiati, and G. Jacovitti. Cumulant series expansion of hybrid nonlinear moments of $\mathrm{n}$ variates. IEEE Transactions on Signal Processing, 41(1):486-489, 1993.

J. Schoukens, R. Pintelon, T. Dobrowiecki, and Y. Rolain. Identification of linear systems with nonlinear distortions. In Preprints of the 13th IFAC Symposium on System Identification, pages 1761-1772, Rotterdam, The Netherlands, August 2003.

J. Schoukens, J. Swevers, R. Pintelon, and H. Van der Auweraer. Excitation design for FRF measurements in the presence of nonlinear distortions. $M e-$ chanical Systems and Signal Processing, 18(4):727-738, 2004.

N. Wiener. Extrapolation, Interpolation and Smoothing of Stationary Time Series. Technology Press and Wiley, New York, 1949. 


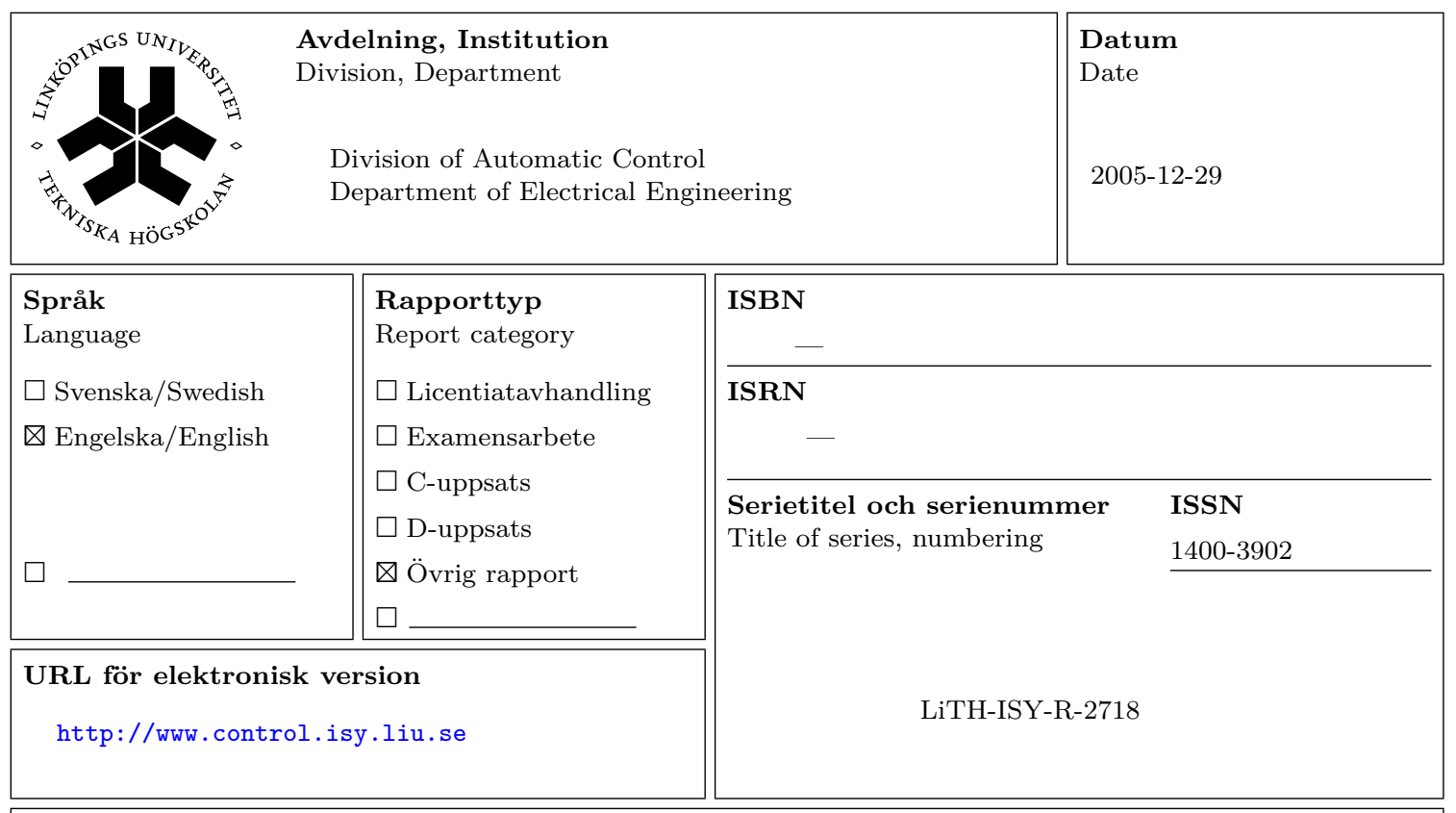

Titel Linear Approximations of Nonlinear FIR Systems for Separable Input Processes

Title

Författare Martin Enqvist, Lennart Ljung

Author

\section{Sammanfattning}

Abstract

Nonlinear systems can be approximated by linear time-invariant (LTI) models in many ways. Here, LTI models that are optimal approximations in the mean-square error sense are analyzed. A necessary and sufficient condition on the input signal for the optimal LTI approximation of an arbitrary nonlinear finite impulse response (NFIR) system to be a linear finite impulse response (FIR) model is presented. This condition says that the input should be separable of a certain order, i.e., that certain conditional expectations should be linear. For the special case of Gaussian input signals, this condition is closely related to a generalized version of Bussgang's classic theorem about static nonlinearities. It is shown that this generalized theorem can be used for structure identification and for identification of generalized Wiener-Hammerstein systems. 